\title{
On the use of dexamethasone-loaded liposomes to induce the osteogenic differentiation of human mesenchymal stem cells
}

\author{
Nelson Monteiro ${ }^{1,2}$, Albino Martins ${ }^{1,2}$, Diana Ribeiro ${ }^{1,2}$, Susana Faria ${ }^{3}$, Nuno A. Fonseca ${ }^{4}$, \\ João N. Moreira ${ }^{4}$, Rui L. Reis ${ }^{1,2}$ and Nuno M. Neves ${ }^{1,2 *}$ \\ ${ }^{1} 3$ Bs Research Group - Biomaterials, Biodegradables and Biomimetics, Department of Polymer Engineering, University of Minho, \\ Headquarters of the European Institute of Excellence on Tissue Engineering and Regenerative Medicine, AvePark, Zona Industrial da \\ Gandra S. Cláudio do Barco, 4806-909 Caldas das Taipas, Guimarães, Portugal \\ ${ }^{2}$ ICVS/3Bs, PT Government Associated Laboratory, Braga, Guimarães, Portugal \\ ${ }^{3}$ Research Centre Officina Mathematical, Department of Mathematics for Science and Technology, University of Minho, Campus de Azurém, \\ 4800-058 Guimarães, Portugal \\ ${ }^{4}$ Centre for Neurosciences and Cell Biology (CNC), Faculty of Pharmacy of the University of Coimbra (FFUC), 3000 Coimbra, Portugal
}

\begin{abstract}
Stem cells have received considerable attention by the scientific community because of their potential for tissue engineering and regenerative medicine. The most frequently used method to promote their differentiation is supplementation of the in vitro culture medium with growth/differentiation factors (GDFs). The limitations of that strategy caused by the short half-life of GDFs limit its efficacy in vivo and consequently its clinical use. Thus, the development of new concepts that enable the bioactivity and bioavailability of GDFs to be protected, both in vitro and in vivo, is very relevant. Nanoparticlebased drug delivery systems can be injected, protect the GDFs and enable spatiotemporal release kinetics to be controlled. Liposomes are well-established nanodelivery devices presenting significant advantages, viz. a high load-carrying capacity, relative safety and easy production, and a versatile nature in terms of possible formulations and surface functionalization. The main objective of the present study was to optimize the formulation of liposomes to encapsulate dexamethasone (Dex). Our results showed that the optimized Dex-loaded liposomes do not have any cytotoxic effect on human bone marrow-derived mesenchymal stem cells (hBMSCs). More importantly, they were able to promote an earlier induction of differentiation of hBMSCs into the osteogenic lineage, as demonstrated by the expression of osteoblastic markers, both phenotypically and genotypically. We concluded that Dex-loaded liposomes represent a viable nanoparticle strategy with enhanced safety and efficacy for tissue engineering and regenerative medicine. Copyright (c) 2013 John Wiley \& Sons, Ltd.
\end{abstract}

Received 21 May 2013; Accepted 24 July 2013

Keywords nanoparticle; liposome; delivery system; bioactive agent; dexamethasone; stem cell differentiation

\section{Introduction}

The advent of nanoscience and nanotechnology in the last 20 years enabled the development of nanoscale devices

\footnotetext{
*Correspondence to: N. M. Neves, 3Bs Research Group - Biomaterials, Biodegradables and Biomimetics, Department of Polymer Engineering, University of Minho, Headquarters of the European Institute of Excellence on Tissue Engineering and Regenerative Medicine, AvePark, Zona Industrial da Gandra S. Cláudio do Barco, 4806-909 Caldas das Taipas, Guimarães, Portugal. E-mail: nuno@dep.uminho.pt
}

that may interact with biological systems at the molecular level. Those devices may be designed to stimulate, respond to and interact with target cells and tissues at the subcellular scale of these physiological events. In this way it is possible to influence these events locally and to minimize the undesirable side-effects that are common in the most traditional systemic strategies (Kim et al., 2010). Nanoparticles that are currently in clinical use as drug-release systems or as imaging devices (Kim et al., 2010) are examples of such devices. As drug-delivery systems, they allow drugs to be released specifically at the intended site of action (Gabizon et al., 1998; Goldberg 
et al., 2007; Tan et al., 2012). Other nanometric drugrelease systems include liposomes, dendrimers and polymeric systems (Santo et al., 2010; Shi et al., 2010; Mickova et al., 2012). The nanoparticle-based systems have the advantage of diffusing through cytoplasmatic cell membranes, allowing cell activityto be controlled or modified. Recently, our group has proposed a strategy based on tissue-engineering principles and using dendrimer nanoparticles (CMCht/PAMAM), which has shown capacity to enable the intracellular release of dexamethasone (Dex; an osteogenic differentiation factor) (Oliveira et al., 2008, 2009). The local release of Dex was also studied in other devices, i.e. starch-based microparticles (SPCL) (Balmayor et al., 2008, 2009a, 2009b) and nanofibres (Martins et al., 2010), showing consistent positive efficacy in driving the osteogenic differentiation of stem cells.

Considering our background in the development of polymer-based nano- and microparticles as drug-release systems, we proposed the development of lipid-based nanoparticles or liposomes for the development of cellbased therapies. Liposomes are well-established carrier systems (Pjanović et al., 2010), enabling the encapsulation of both hydrophilic and lipophilic compounds (Bhardwaj and Burgess, 2010); the stability of the liposomes may be enhanced by adding cholesterol in the formulation (Lee et al., 2005). The liposomes can also be sterically stabilized by using polymer additives, e.g. PEG, into the formulation. These liposomes are very stable both during storage and upon injection into the blood plasma (Loughrey et al., 1993; Song et al., 2009). Liposomes present significant advantages over other nanoparticle-based drug-release systems, such as a high load-carrying capacity (Lee et al., 2005; Liu and Park, 2010), low cytotoxicity (Moreira et al., 2002) and a versatile structure in terms of possible formulation and functionalization (Drummond et al., 1999). Various bioactive molecules have been loaded in lipid-based systems, using a variety of preparation methods (Almeida and Souto, 2007; Gomes-da-Silva et al., 2012; Moura et al., 2012). The liposomes can be administrated by many routes, such as intravenously (Chono et al., 2006), orally (Li et al., 2003), by inhalation (Chen et al., 2012), by local injection (Hegeman et al., 2011) or by ocular administration (Diebold et al., 2007).

Human mesenchymal stem cells (hMSCs) are adult stem cells with great potential in different therapeutic contexts, but particularly for tissue engineering and regenerative medicine. Those cells can be isolated from adult tissue sources such as bone marrow, cord blood, placenta, adipose and dermal tissues, synovial fluid, deciduous teeth and amniotic fluid (Engler et al., 2006; Katayama et al., 2006). Moreover, they have the potential for expansion and the ability to differentiate into various mesodermal tissues, e.g. bone, cartilage, fat, muscle and other connective tissues (Huang et al., 2005; Martins et al., 2010). hMSCs may provide a useful model for evaluating the multiple factors responsible for the stepwise progression of cells from undifferentiated precursors to secretory osteoblasts and, eventually, terminally differentiated osteocytes (Jaiswal et al., 1997; Park et al., 2007).
The regulation of stem cell differentiation by drugs and growth factors might enable the fabrication of therapeutic materials for the delivery of stem cells that are simpler to use, cost-effective and more easily controlled than the delivery systems currently available (Park et al., 2011). The combination of nanoparticles and hMSCs presents a new opportunity to develop advanced therapy medicinal products (Park et al., 2007; Oliveira et al., 2009, 2010).

To our best knowledge, no previous reports published in the literature have explored the possibility of using Dex-releasing liposomes as a new method to induce the differentiation of MSCs, either in vitro or in vivo. In this study we aimed to optimize the incorporation of an osteogenic differentiation factor, Dex, into liposomes. Furthermore, we studied the effect of Dex released from the liposomes on the viability, proliferation, protein synthesis and differentiation of hBMSCs. By using Dex-loaded liposomes, we expected to release this bioactive agent near to, or even to internalize it into, the hBMSCs.

\section{Experimental}

\subsection{Development of Dex-loaded liposomes}

\subsubsection{Materials}

Chloroform, dexamethasone (Dex) ammonium molybdate, Fiske-Subbarow reducer and sepharose CL4B, HEPES buffer solution (HBS), were reagent grade and purchased from Sigma-Aldrich. The lipids L- $\alpha$-phosphatidylethanolamine- $N$-(lissamine rhodamine B sulphonyl; ammonium salt, egg-transphosphatidylated, chicken) (PE-Rho), 1,2distearoyl-sn-glycero-3-phosphoethanolamine- $N$-[methoxy (polyethylene glycol)-2000] (ammonium salt; DSPE-PEG), cholesterol (ovine wool, > 98\%; Chol) and L- $\alpha$-phosphatidylcholine, hydrogenated (Soy; HSPC) were purchased from Avanti Polar Lipids. Dialysis tubing cellulose membrane (100-500 molecular weight cut off (MWCO), $10 \mathrm{~mm}$ flat width) was purchased from Spectrum Laboratories. All the materials were used as received.

\subsubsection{Production and characterization of Dex-loaded liposomes}

Table 1 shows the liposome formulations used in this study. Formulations A-D were used to study encapsulation efficiency and the effect of adding Chol and Dex to the liposome bilayer; formulation $\mathrm{E}$ was used to perform the release study of the liposomes from the dialysis tubing; and formulation $\mathrm{F}$ was used for biological assays.

Lipids and Dex were mixed in a round-bottomed flask, withdrawing appropriate amounts of each lipid from the stock solutions (15 mM total lipid), in the proportions described for each type of liposomal formulation. The solvent chloroform was slowly evaporated using a gentle steam of nitrogen. The obtained dry film was dispersed using vortex agitation with HBS, keeping the temperature 
Table 1. Liposome formulations (values expressed as a molar ratio)

\begin{tabular}{lccccc}
\hline Formulation & Dex & HSPC & Chol & DSPE-PEG & PE-Rho \\
\hline A & 0.25 & 2 & - & - & - \\
B & 0.50 & 2 & - & - & - \\
C & 0.25 & 2 & 0.1 & - & - \\
D & 0.25 & 2 & 1 & - & - \\
E & 0.25 & 2 & 0.1 & 0.1 & - \\
F & 0.25 & 2 & 0.1 & 0.1 & 0.02 \\
\hline
\end{tabular}

of the hydrating medium above the gel-liquid crystal transition temperature $\left(T_{c}=52^{\circ} \mathrm{C}\right)$. The multilamellar liposomal suspension was extruded (using an Avanti Mini-Extruder) at $T>T_{c}$ through a porous polycarbonate membrane $(100 \mathrm{~nm})$. The system was used to force the solution back and forward (21 times), resulting in unilamellar liposomes. Non-encapsulated Dex was removed from the solution by column chromatography (Sepharose CL4B, Sigma-Aldrich), using an isocratic elution with HBS.

Particle size distribution and $\zeta$-potential were determined by dynamic light scattering (Zetasizer Nanoseries ZS, Malvern Instruments). Liposome morphology was analysed by scanning electron microscopy (SEM) and scanning transmission electron microscopy (STEM; NanoSEM Nova 200). The SEM supports were gold-coated (Cressington 208 HR) before dropping the liposomal solution onto them. After drying at room temperature, the SEM supports were coated again with a thin film of gold and palladium (12 $\mathrm{nm})$.

\subsubsection{Dex loading efficiency into liposomes}

The Dex loading efficiency was analysed, using formulations A-D, by quantifying Dex and lipid content. Dex concentration was determined by UV-VIS spectroscopy at 247 nm (Synergie HT), using Dex-free liposomes as blanks. The total lipid concentration was assessed by the Bartlett colorimetric assay, as described elsewhere (Torchilin and Weissig, 2003). The principle of the Bartlett assay is based on the colorimetric determination of inorganic phosphate. The phospholipid content of liposomes can be determined after destruction of the phospholipid with perchloric acid to inorganic phosphate. The inorganic phosphate is converted to phospho-molybdic acid by the addition of ammonium molybdate, which is reduced to a blue complex by 4-amino-2-naphthyl-4-sulphonic acid during heating. This compound was determined colorimetrically at $830 \mathrm{~nm}$ (Synergie HT).

The ability of the liposomal vesicles to incorporate the drug was evaluated by calculating the system payload $(P L)$ from equation (1). The encapsulation efficiency $(E E)$ was calculated as the final $P L\left(P L_{f}\right)$ per initial $P L$ $\left(P L_{i}\right)$ of Dex and HSPC from equation (2).

$P L=$ amount of Dex $(\mathrm{mol}) /$ amount of $\operatorname{HSPC}(\mathrm{mol})$

$$
E E(\%)=P L_{f} / P L_{i}^{\prime} \times 100
$$

\subsubsection{Dex release kinetics from the liposomes}

The release of Dex from the loaded liposomes was studied using a dialysis method. Dialysis cellulose tubes (100-500 MWCO, Spectrum Laboratories) were rinsed with distilled water for 1 week prior to their use. The tube ends were closed with Teflon tape and Nylon thread and tested for leakage. $1 \mathrm{ml}$ of each liposomal solution (formulation E, $\sim 17 \%$ EE) was added to the dialysis tubes and fully immersed in $10 \mathrm{ml}$ phosphate-buffered saline (PBS) as the release medium. Control tubes were assembled using the same procedure with Dex-free liposomes. The solutions were maintained at $37^{\circ} \mathrm{C}$ and $60 \mathrm{rpm}$ for 21 days. For each time point, an aliquot of $1 \mathrm{ml}$ was collected from the solution and analysed by UV-VIS spectroscopy at $247 \mathrm{~nm}$ (Synergie HT). The same volume of fresh PBS was replaced to keep the total volume of the release medium at $10 \mathrm{ml}$. The Dex concentration in the release medium was determined based on a standard curve in the range 0-0.125 mM Dex and the measured values used to calculate the cumulative release. The experiments were done in triplicate.

\subsection{Biological assays}

\subsubsection{Expansion, seeding and osteogenic differentiation of hBMSCS}

hBMSCs were isolated from bone marrow aspirates collected under informed consent from patients undergoing knee arthroplasty at the Hospital de Braga, Portugal. Samples were collected from a 58 year-old female donor, isolated, expanded and cryopreserved until further use. hBMSCs were isolated and characterized according to the method established by Delorme and Charbord (2007). Briefly, plastic adherent fractions of marrow cells characterized by a spindle-shaped morphology and colony-forming unit (CFU) capacity; expression of surface antigens such as CD29, CD73, CD90 and CD105, while negative for haematopoietic markers such as CD34 and CD45 and by their differentiation potential into the osteogenic, chondrogenic and adipogenic lineages were used; all the antibodies were purchased from BD Pharmingen and the hBMSCs analysed on a FACS Calibur, BD Biosciences. hBMSCs were expanded in basal medium consisting of $\alpha$-minimal essential medium ( $\alpha$-MEM; Gibco, UK) supplemented with $10 \%$ heat-inactivated fetal bovine serum (FBS; BiochromAG, Germany) and 1\% antibioticantimyotic solution (final concentration of penicillin $100 \mathrm{U} / \mathrm{ml}$ and streptomycin $100 \mathrm{mg} / \mathrm{ml}$; Gibco, UK). The cells were cultured at $37^{\circ} \mathrm{C}$ in a humidified atmosphere of $5 \% \mathrm{CO}_{2}$. The liposomal solutions (formulation $\mathrm{F}$, $\sim 17 \%$ EE) were sterilized by $0.22 \mu \mathrm{m}$ filtering, and $0.5 \mu \mathrm{l}$ LipDex was added to $1 \mathrm{ml}$ culture medium. Confluent hBMSCs at passage 4 were harvested for seeding onto 24 -well plates at a density of $1 \times 10^{5}$ cells. After $24 \mathrm{~h}$ of incubation in basal medium, the hBMSCs were cultured on Dex-free osteogenic differentiation medium (basal medium 
supplemented with $50 \mu \mathrm{g} / \mathrm{ml}$ ascorbic acid and $10 \mathrm{~mm}$ $\beta$-glycerophosphate) in the presence of Dex-loaded liposomes. The control condition was cultured on standard osteogenic differentiation medium (basal medium supplemented with $50 \mu \mathrm{g} / \mathrm{ml}$ ascorbic acid, $10 \mathrm{~mm}$ $\beta$-glycerophosphate and $10^{-7} \mathrm{M}$ Dex). The cells were retrieved at predefined culture times (7, 14 and 21 days). The experiments were performed twice, independently, with sample triplicates, with hBMSCs from the same donor.

\subsubsection{Cell viability and proliferation assessment}

Cell viability for each culture condition and time point was determined using the CellTiter 96 $\mathrm{R}$ AQueous One Solution Cell Proliferation Assay (Promega, USA), according to the manufacturer's instructions. This assay is based on the bioreduction of a tetrazolium compound, 3-(4,5dimethylthiazol-2-yl)-5-(3-carboxymethoxyphenyl)-2(4-sulphofenyl)-2H-tetrazolium (MTS), into a water-soluble brown formazan product. The absorbance was measured at $490 \mathrm{~nm}$ in a microplate reader (SynergieHT, Bio-Tek, USA), being related to the quantity of formazan product and directly proportional to the number of living cells in culture. Four samples/time point were quantified.

Cell proliferation was quantified by the total amount of double-stranded DNA along the culture time. Quantification was performed using the Quant-i ${ }^{\mathrm{TM}}$ Pico-Green dsDNA Assay Kit (Invitrogen ${ }^{\mathrm{TM}}$, Molecular Probes ${ }^{\mathrm{TM}}$, OR, USA), according to the manufacturer's instructions. Briefly, cells in the construct were lysed by osmotic and thermal shock and the supernatant used for the DNA quantification assay. The fluorescence of the dye was measured at an excitation wavelength of $485 / 20 \mathrm{~nm}$ and at an emission wavelength of $528 / 20 \mathrm{~nm}$ in a microplate reader (Synergie HT, BioTek; USA). Quadruplicates were made for each sample and culture time. The DNA concentration for each sample was calculated using a standard curve (DNA concentration in the range $0.0-1.5 \mu \mathrm{g} / \mathrm{ml}$ ), relating DNA quantity to the fluorescence intensity.

All data concerning cell viability, proliferation and ALP activity were independently measured and normalized against the cell number for each sample. For that, a standard calibration curve was constructed, using known hBMSCs cell numbers at passage 4 , in the range $0-5 \times 10^{5}$ cells $(n=12)$. The dsDNA concentration of these samples was determined according to the Quant-iT ${ }^{\mathrm{TM}}$ PicoGreen dsDNA reagent previously described. The following equation was obtained:

$$
y=0.0054 x+86.68
$$

where $R^{2}=0.998, y$ is the measured fluorescence value and $x$ is the cell number, and used to estimate the cell number for each sample.

\subsubsection{Alkaline phosphatase and total protein quantification}

The concentration of alkaline phosphatase (ALP) was determined for all culture periods, using the lysates used for DNA quantification. Briefly, the ALP quantity was assessed using the $p$-nitrophenol assay, in which 4-nitrophenyl phosphate disodium salt hexahydrate (Sigma, USA) is hydrolysed by intracellular ALP at a temperature of $37^{\circ} \mathrm{C}$ in an alkaline buffer solution (1.5 M and $\mathrm{pH} 10.5$; Sigma) to form free, yellow $p$-nitrophenol. The reaction was stopped by the addition of $0.3 \mathrm{M} \mathrm{NaOH}$ (Panreac Quimica, Spain) and the absorbance read at $405 \mathrm{~nm}$ in a microplate reader (Bio-Tek). Standards were prepared with $10 \mathrm{~mm}$ p-nitrophenol (pNP; Sigma, USA) solution to obtain a standard curve in the range 0-250 $\mu \mathrm{M}$. Quadruplicates of each sample and standard were made, and the ALP concentrations read off the standard curve.

For the quantification of total protein synthesized by the hBMSCs in culture, the Micro BCA ${ }^{\mathrm{TM}}$ Protein Assay kit (Thermo Scientific, Pierce; Rockford, USA) was used according to the manufacturer's instructions. This is a colorimetric detection and quantification method which utilizes bicinchoninic acid (BCA) as the detection reagent for $\mathrm{Cu}^{+1}$ formed when $\mathrm{Cu}^{+2}$ is reduced by the protein in an alkaline environment. Quadruplicates of lysed cells per culture time were incubated at $37^{\circ} \mathrm{C}$ for $2 \mathrm{~h}$. A purple reaction product was measured at $562 \mathrm{~nm}$ in a microplate reader (Bio-Tek) and calculated based on an albumin standard curve in the range $0-40 \mu \mathrm{g} / \mathrm{ml}$.

\subsubsection{Immunocytochemistry for bone-specific proteins}

At predefined culture times, hBMSCs were fixed in $10 \%$ formalin solution neutral buffer (Sigma-Aldrich, Germany). After an initial washing with PBS, the cells were treated with $0.3 \%$ hydrogen peroxide in methanol for $30 \mathrm{~min}$ to inactivate the endogenous peroxidases. After washing with PBS, the cells were blocked with 3\% BSA solution for $20 \mathrm{~min}$ at room temperature to avoid non-specific reactions. Immunocytochemistry was performed using a rabbit polyclonal antibody against osteopontin (Abcam, Cambridge, UK; dilution 1:1500) and a mouse monoclonal antibody against osteocalcin (clone OC4-30, Abcam; dilution 1:100), and an incubation period of $2 \mathrm{~h}$ at room temperature. After washing in PBS, the samples were incubated for $30 \mathrm{~min}$ with biotinylated secondary antibody anti-rabbit/mouse IgG (from the R.T.U. Vectastain $®$ Universal Elite $A B C$ kit, Vector Laboratories, Burlingame, CA, USA), followed by incubation with streptavidin-peroxidase complex (Elite $\AA$ ABC Reagent; Vector Laboratories). The immune reaction was visualized using DAB as a chromogen (DAB Substrate Reagent from Peroxidase Substract Kit, Vector Laboratories) and counterstained with Harris's haematoxylin (Bio-Optica, Milano, Italy). The slides were observed under an optical microscope (BX61, Olympus, Germany) and images captured by a digital camera (DP70, Olympus).

\subsubsection{RNA isolation and real-time quantitative polymerase chain reaction}

At each culture time point the hBMSCs were washed with PBS, immersed in Tri $\circledR$ reagent (Sigma-Aldrich) and stored 
at $-80^{\circ} \mathrm{C}$ until further use. Proteins were removed with chloroform:isoamylalcohol (BioChemica, AppliChem, Germany) extraction and the RNA pellets were washed once with 2-propanol (Sigma-Aldrich) and once with 70\% ethanol (Panreac, Spain). The total RNA pellets were reconstituted in RNase-free water (Gibco, Invitrogen, UK). Determination of the RNA concentration for each replica (quadruplicates of each condition per time point) was performed by microspectrophotometry (NanoDrop 1000, Thermo Scientific, USA).

Reverse-transcriptase (RT)-polymerase chain reaction (PCR) was performed according to the protocol from iScript $^{\mathrm{TM}}$ cDNA synthesis kit (Quanta BioSciences ${ }^{\mathrm{TM}}$, Gaithersburg, MD, USA). Briefly, a reaction mixture consisting of $1 \times$ iScript Reaction Mix, $1 \mu 1$ iScript Reverse Transcriptase, 100-150 ng RNA template and nuclease-free water was prepared in $20 \mu \mathrm{l}$ total volume. The single-strand cDNA synthesis occurred by incubating the complete reaction mixture for $5 \mathrm{~min}$ at $22^{\circ} \mathrm{C}$, followed by $30 \mathrm{~min}$ at $42^{\circ} \mathrm{C}$, and terminated by an incubation at $85^{\circ} \mathrm{C}$ for $5 \mathrm{~min}$.

Amplification of the target cDNA for real-time PCR quantification were performed according to the manufacturer's instructions, using $2 \mu \mathrm{l}$ RT cDNA product, $250 \mathrm{~nm}$ each primer, $1 \times$ PerfeCta ${ }^{\circledR}$ SYBR $\AA$ Green FasterMix ${ }^{\circledR}$ (Quanta BioSciences) and nuclease-free water in a final volume of $25 \mu \mathrm{l}$. Forty-four cycles of denaturation $\left(95^{\circ} \mathrm{C}\right.$, $10 \mathrm{~s}$ ), annealing (temperature dependent on the gene, $30 \mathrm{~s})$ and extension $\left(72^{\circ} \mathrm{C}, 30 \mathrm{~s}\right)$ were carried out in a Mastercycler Epgradient S Realplex Thermocycler (Eppendorf, Hamburg, Germany) for all genes. The transcript expression data were normalized to the housekeeping gene glyceraldehyde-3-phosphate-dehydrogenase (GAPDH) and the quantification performed according to the Livak $\left(2^{-\triangle \Delta C T}\right)$ method, considering the tissue culture polystyrene (TCPS)_Osteo culture condition as the calibrator.

\subsubsection{Statistical analysis}

Data were statistically analysed using IBM SPSS software v. 20 (SPSS Inc., USA). We first applied the Shapiro-Wilk test to test the assumption of Normality and the results showed that the data were not following a Normal distribution. Consequently, the non-parametric Mann-Whitney $\mathrm{U}$ (to test data differences between the TCPS_Osteo and the TCPS_LipDex conditions) and Kruskal-Wallis (to test data differences between culture days for each condition) methods were applied. $p<0.01$ was considered statistically significant.

\section{Results}

\subsection{Development and characterization of liposomes}

In this study we prepared six different liposomal formulations, including four conventional liposome (CL) formulations (A-D) and two sterically stabilized liposome (SSL) formulations with DSPE-PEG (E) and SSL with DSPE-PEG and PE-Rho (F). The CL and SSL formulations differed in the presence of a polymer coating at the surface of the liposome, most commonly polyethylene glycol (PEG).

\subsubsection{Drug encapsulation}

$P L$ and EE are presented in Table 2. Analysing the data, it is demonstrated that the increase of Dex concentration significantly reduces its encapsulation efficiency $(p<0.001)$, while the observed variations in the system PL are not significant. These results reveal that the maximum encapsulation was reached with a Dex:HSPC ratio of $0.25: 2$. On increasing the Chol proportion from 0.0 to 0.1 no significant variations were observed in the system PL; increasing Chol to 1.0 significantly reduced the $P L$ and $E E$ to near-zero encapsulation. The encapsulation of Dex was higher when no Chol was added (49 molecules Dex incorporated/100 molecules lipid). With the data obtained in this study, a compromise was obtained with the formulation 0.25:2.0:0.1 (Dex:HSPC:Chol) that was subsequently used in the Dex release study.

\subsubsection{Liposome size and $\zeta$-potential}

Table 3 presents the size and $\zeta$-potential of each of the produced liposome formulations. The incorporation of Chol into the liposomes did not result in any significant change in the mean diameter of the extruded Dex-loaded liposomes. The $\zeta$ - potential of the CL was close to neutrality and no significant difference was observed due to incorporation of Chol and Dex (formulations A-D); however, the $\zeta$-potential increased for the PEGylated liposomes (formulations $\mathrm{E}$ and F). The particle size distribution of the liposomes prepared in this study showed a monodisperse distribution and there was no significant change in particle size of the CLs and DSPE-PEG-coated liposomes.

Table 2. Effect of varying the Dex and Chol concentrations in Dex PL and EE

\begin{tabular}{lccr}
\hline Formulation & Dex:HSPC:Chol & PL $(\mathrm{mol} / \mathrm{mol})$ & EE $(\%)$ \\
\hline A & $0.25: 2: 0.0$ & $0.49 \pm 0.10$ & $26.38 \pm 3.89$ \\
B & $0.50: 2: 0.0$ & $0.41 \pm 0.01$ & $11.09 \pm 2.50$ \\
C & $0.25: 2: 0.1$ & $0.31 \pm 0.06$ & $17.01 \pm 5.21$ \\
D & $0.25: 2: 1.0$ & $0.00 \pm 0.00$ & $0.27 \pm 0.20$ \\
\hline
\end{tabular}

Table 3. $\zeta$-Potential and mean particle diameter of Dex-loaded liposomes

\begin{tabular}{lcl}
\hline Formulation & $\zeta$-Potential & \multicolumn{1}{c}{ Size $(\mathrm{nm})$} \\
\hline A & $-0.04 \pm 2.68$ & $123.53 \pm 6.60$ \\
B & $-2.69 \pm 1.00$ & $135.63 \pm 10.25$ \\
C & $-5.84 \pm 4.54$ & $122.90 \pm 3.76$ \\
D & $-1.43 \pm 1.56$ & $122.23 \pm 19.18$ \\
E & $-20.00 \pm 1.25$ & $113.50 \pm 5.11$ \\
F & $-22.50 \pm 3.40$ & $103.70 \pm 17.74$ \\
\hline
\end{tabular}




\subsubsection{STEM and SEM of Dex-loaded liposomes}

Figure 1 shows STEM and SEM images of the Dex-loaded liposomes (formulations $\mathrm{C}$ and $\mathrm{E}$ ). The liposomes in formulation $C$ (Figure 1A) aggregated more than the liposomes in formulation E with DSPE-PEG (Figure 1B) at the higher liposome concentration. Analysing the SEM image of non-coated liposomes prepared with formulation C (Figure 1C), it is clear that the liposomes were fused to each other and were spread and flattened at the support surface. From Figure 1D, it was observed that the DSPE-PEG liposomes presented a nano-scale size and spherical shape.

\subsubsection{Dex release from liposomes}

The release profile of Dex from the DSPE-PEG-coated liposomes was performed in dialysis tubes for 21 days, using formulation E. This time frame was selected in accordance with the culture time usually required to obtain a complete osteogenic differentiation of MSCs in vitro. The release profile of Dex (Figure 2) showed an initial burst release within $12 \mathrm{~h}$. Following the initial release, a slower release was observed until day 6. Afterwards, Dex continued to be released at a slower but steady rate until day 21 .

\subsection{Biological activity}

\subsubsection{Cell viability and proliferation assessment}

Initially, the influence of Dex-loaded liposomes over hBMSCs viability and proliferation was assessed using

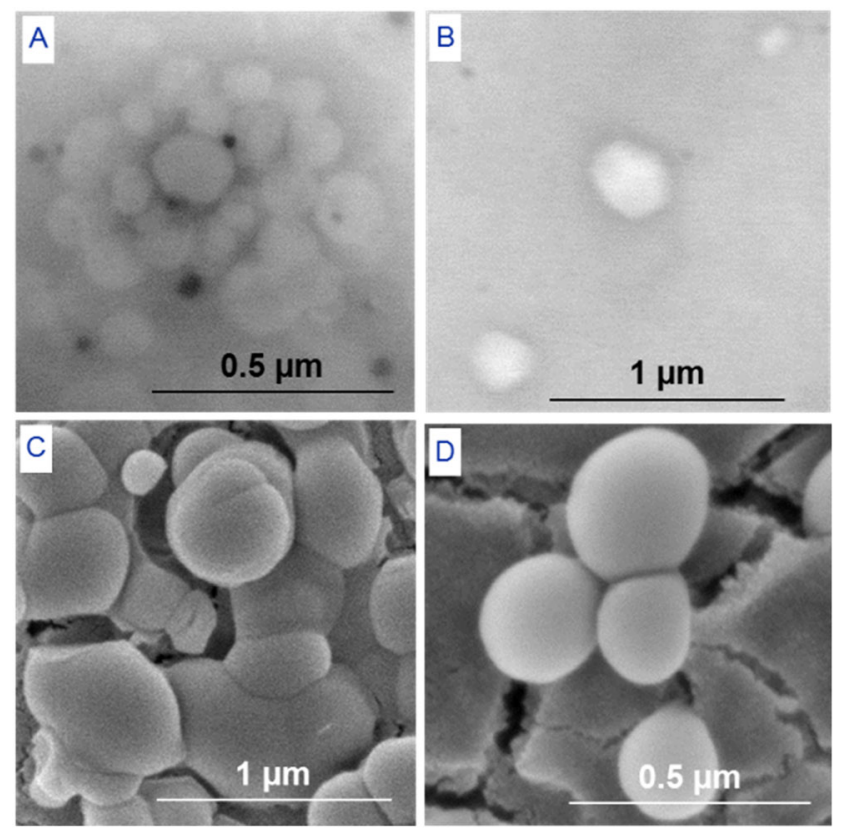

Figure 1. STEM and SEM images of Dex-loaded liposomes. STEM: (A) formulation C; (B) formulation E. SEM: (C) uncoated liposomes, formulation C; (D) DSPE-PEG-coated liposomes, formulation $\mathrm{E}$

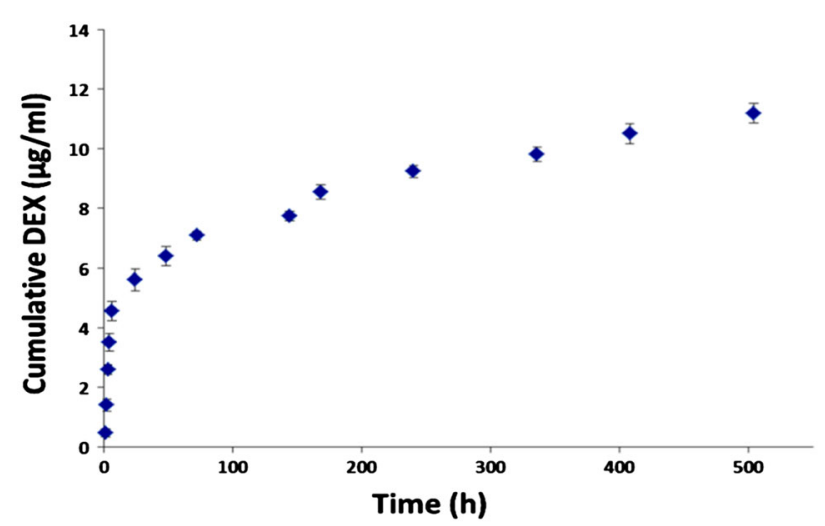

Figure 2. In vitro cumulative Dex release from DSPE-PEG liposomes (formulation E)

standard cell biology protocols, i.e. MTS and PicoGreen assays, respectively. In terms of cell viability, no significant differences were found between the standard TCPS_Osteo and the TCPS_LipDex culture conditions for days 7 (Mann-Whitney U-test, $p=0.409$ ) and 21 (Mann-Whitney U-test, $p=0.766)$. TCPS_Osteo only displayed a significantly higher cell viability value than TCPS_LipDex for day 14 of hBMSCs culture (Mann-Whitney U-test, $p<0.001$ ) (Figure 3). Additionally, no significant differences were observed over time in standard TCPS_Osteo (Kruskal-Wallis test, $p=0.022$ ) and in the TCPS_LipDex test condition (Kruskal-Wallis test, $p=0.067$ ).

In terms of DNA concentration, no significant difference between TCPS_Osteo and TCPS_Lip was found on days 7 (Mann-Whitney U-test, $p=0.932$ ), 14 (Mann-Whitney U-test, $p=0.755$ ) and 21 (Mann-Whitney U-test, $p=0.989$ ) (Figure 4). No significant differences were found between culture times in TCPS_Osteo (Kruskal-Wallis test, $p=0.945$ ) and TCPS_Lip (Kruskal-Wallis test, $p=0.919$ ).

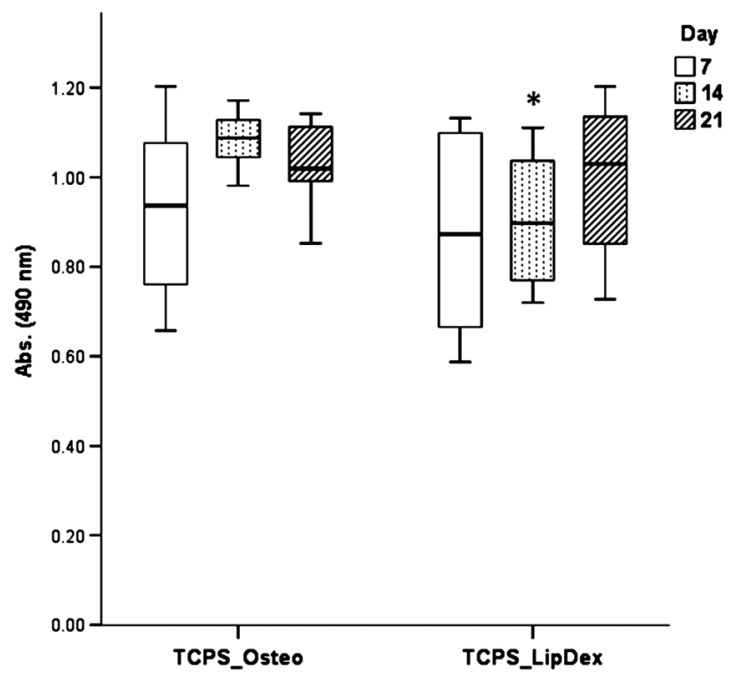

Figure 3. Box plot of hBMSCs viability (MTS assay) cultured on TCPS_Osteo and TCPS_LipDex after 7, 14 and 21 days. Data were analysed non-parametrically by Mann-Whitney U-test (*p 0.01 vs TCPS_Osteo) 


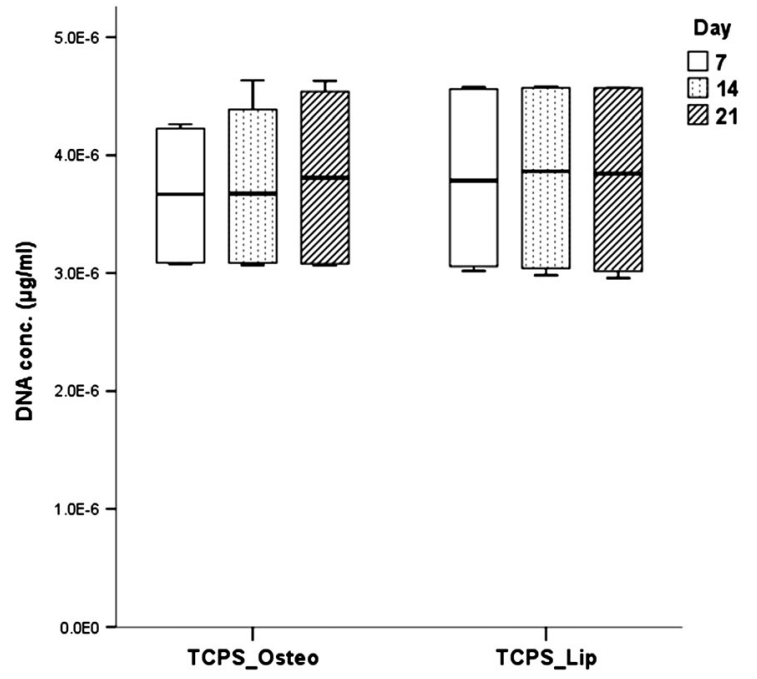

Figure 4. Box plot of the DNA concentration $(\mu \mathrm{g} / \mathrm{ml})$ in TCPS_Osteo and TCPS_Lip after 7, 14 and 21 days of culture. Data were analysed non-parametrically by Mann-Whitney U-test

\subsubsection{Alkaline phosphatase activity quantification}

In order to assess the onset of osteoblastic activity of the cultured hBMSCs, the quantification of the enzyme alkaline phosphatase was performed according to a standard method, the $p$-nitrophenol assay.

Importantly, the TCPS_LipDex test condition displayed a significantly higher ALP activity than the standard TCPS_Osteo condition already at day 7 of hBMSCs culture (Mann-Whitney U-test, $p<0.001$ ) (Figure 5). However, no significant differences were found between TCPS_Osteo and TCPS_LipDex for day 14 (Mann-Whitney U-test, $p=0.107$ ) and 21 (Mann-Whitney U-test, $p=0.710$ ) of hBMSCs culture. Additionally, no significant differences were found between culture days in the TCPS_LipDex test condition (Kruskal-Wallis test, $p=0.906$ ). Conversely, in

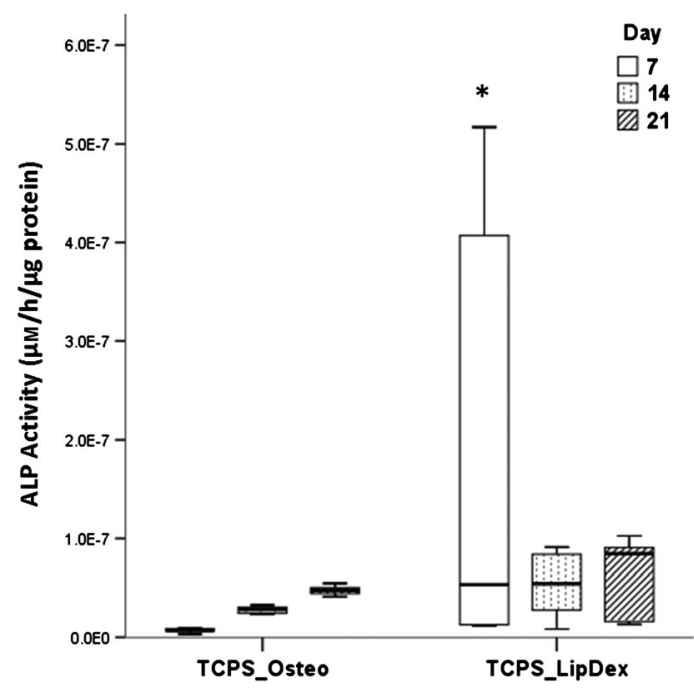

Figure 5. Box plot of ALP activity ( $\mu \mathrm{M} / \mathrm{h} / \mu \mathrm{g}$ protein) in TCPS_Osteo and TCPS_LipDex after 7, 14 and 21 days of culture. Data were analysed non-parametrically by Mann-Whitney U-test ( ${ }^{*} p<0.01$ vs TCPS_Osteo) the standard TCPS_Osteo condition, day 14 displayed a significantly higher ALP activity than day 7 (Kruskal-Wallis test, Tukey's HSD test, $p<0.001$ ), and day 21 displayed a significantly higher ALP activity than days 7 and 14 of hBMSCs culture (Kruskal-Wallis test, Tukey's HSD test, $p<0.001$ ), suggesting that the differentiation takes longer to be induced and follows a more sequential pattern.

\subsubsection{Genotypic and phenotypic expression of earlier osteoblastic markers}

In a first attempt to characterize the expression of the osteoblastic proteins Osteopontin and Osteocalcin, both involved in the homeostasis of bone tissue, an immunolocalization procedure was conducted. As noticed by the strongest intensity of the brownish staining (Figure 6), hBMSCs cultured in the presence of Dex-loaded liposomes showed protein expression of Osteopontin and Osteocalcin earlier in time (i.e. 7 days of culture) than hBMSCs cultured under standard osteogenic differentiation conditions. This protein expression pattern was maintained until day 14 of hBMSCs culture, decreasing for longer time periods (i.e. 21 days) in both culture conditions.

Regarding the quantitative expression of osteoblastic RNA transcripts (Figure 7), the alkaline phosphatase $(A L P)$ gene presented no significant differences between the standard TCPS_Osteo and the TCPS_LipDex culture conditions for days 7 (Mann-Whitney U-test, $p=0.285$ ), 14 (Mann-Whitney U-test, $p=0.285$ ) and 21 (Mann-Whitney U-test, $p=0.068)$. For the osteopontin $(O P)$ gene, also no significant difference between TCPS_Osteo and TCPS_ LipDex was found on days 7 (Mann-Whitney U-test, $p=0.685$ ), 14 (Mann-Whitney U-test, $p=0.845$ ) and 21 (Mann-Whitney U-test, $p=0.285$ ) of hBMSCs culture. For the osteocalcin $(O C N)$ gene, no significant difference between TCPS_Osteo and TCPS_LipDex was found for days 7 (Mann-Whitney U-test, $p=0.685$ ), 14 (Mann-Whitney U-test, $p=0.715$ ) and 21 (Mann-Whitney U-test, $p=0.109$ ). Concerning the expression of the transcriptional factor Runx2, no significant difference between TCPS_Osteo and TCPS_LipDex was found on days 7 (Mann-Whitney U-test, $p=0.465$ ), 14 (Mann-Whitney U-test, $p=0.465$ ) and 21 (Mann-Whitney U-test, $p=0.285$ ). In the Osterix transcriptional factor, no significant differences were also found between TCPS_Osteo and TCPS_LipDex for days 7 (Mann-Whitney U-test, $p=0.465$ ), 14 (Mann-Whitney U-test, $p=0.068$ ) and 21 (Mann-Whitney U-test, $p=0.068$ ) of hBMSCs culture.

\section{Discussion}

\subsection{Development and characterization of Dex-loaded liposomes}

The use of liposomes as a biological drug carrier has several advantages for tissue engineering and regenerative medicine (Kulkarni et al., 2010): liposomes have the great 


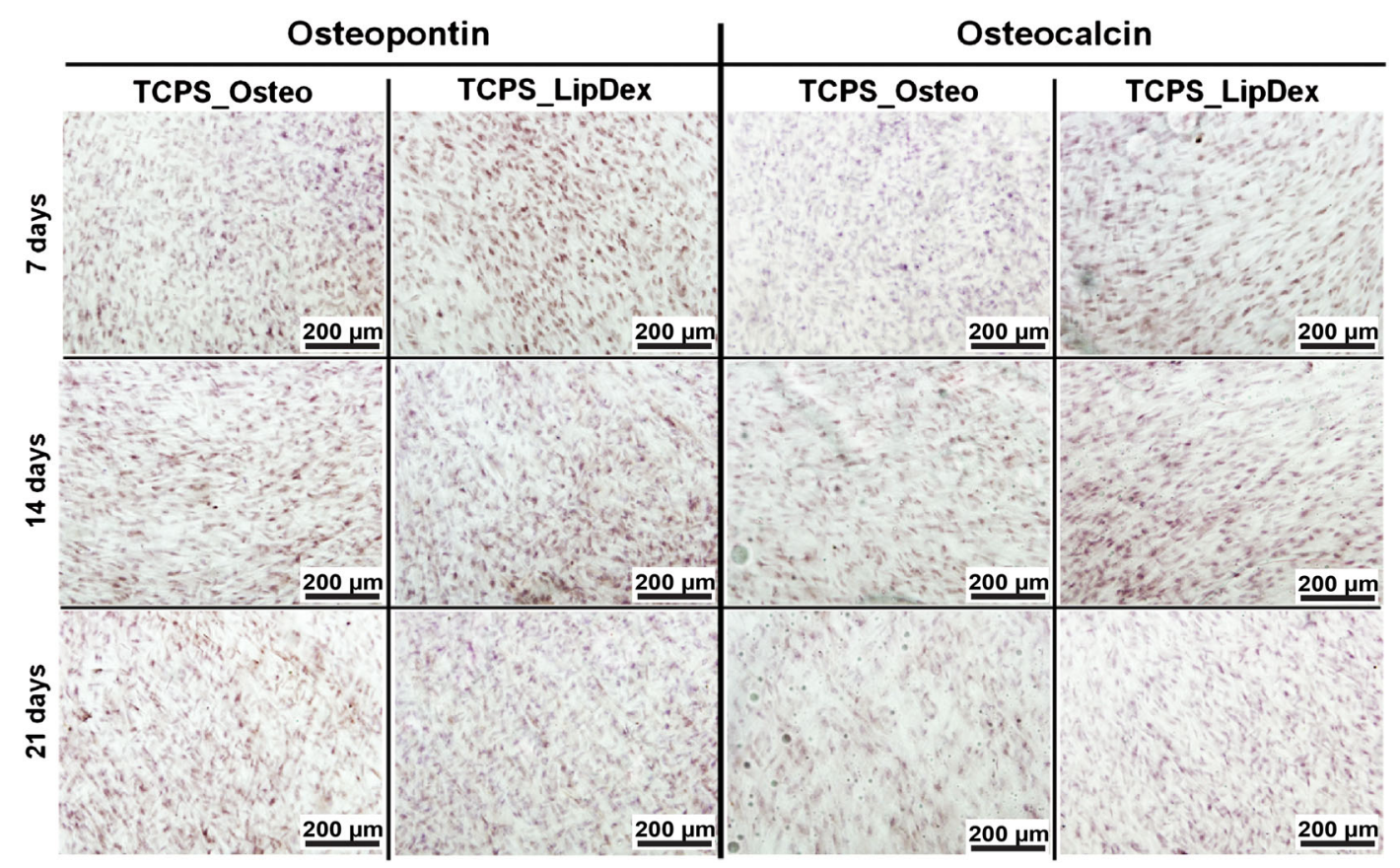

Figure 6. Immunocytochemistry for osteopontin and osteocalcin, expressed by hBMSCs cultured on TCPS_Osteo and TCPS_Lip after 7, 14 and 21 days

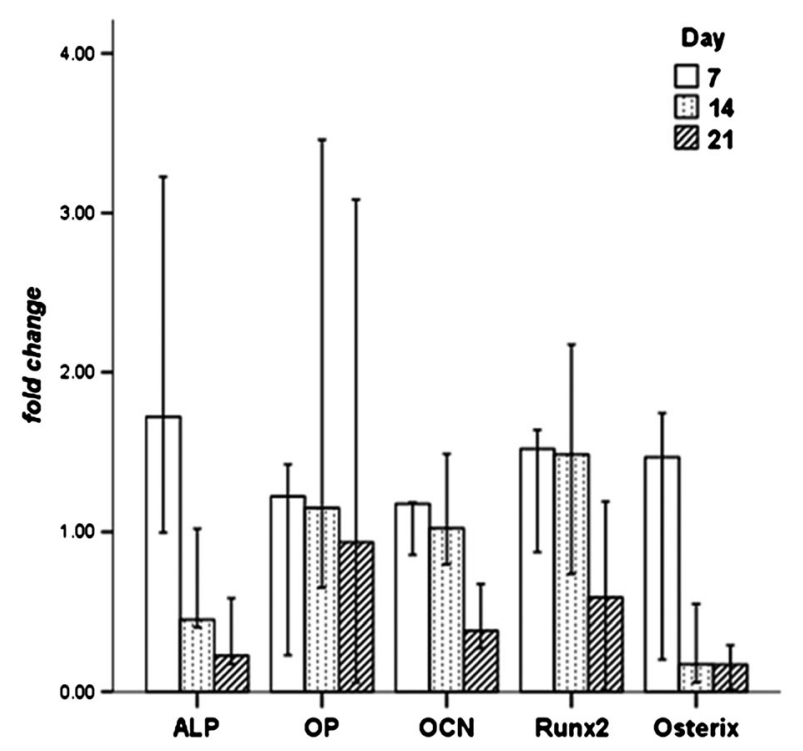

Figure 7. Relative expression of bone-specific transcripts by hBMSCs cultured on Dex-loaded liposomes for 7, 14 and 21 days, normalized to TCPS_Osteo. Data were analysed non-parametrically by Mann-Whitney U-test

advantage of being generally non-toxic for cells; they can be used to encapsulate both hydrophilic and lipophilic growth or differentiation factors, using the bilayer compartment and the inner core for release of the contents directly into the site of action; and it is possible to control the release of growth or differentiation factors by adjusting the liposomal formulation and applying different stimuli, such as $\mathrm{pH}$ (Kale and Torchilin, 2007), temperature (Bhardwaj and Burgess, 2010), ultrasonic waves (Leong-Poi et al., 2007), magnetic fields (Zheng et al., 2009) and light (Wu et al., 2008). A critical design parameter in the formulation of liposomes is the maximum amount of PEG-lipids that can be incorporated into the phospholipid bilayer before its conversion into a micelle (Hristova et al., 1995). This coating provides steric stabilization to the liposomes, which limits opsonization as well as direct interactions with cells, most importantly in the reticuloendothelial system (Allen et al., 1991). Moreover, the liposomes have the advantage of forming more stable formulations and are able to retain the encapsulated drug more efficiently. HSPC was selected because it has a high phase transition temperature, $T_{c}=52^{\circ} \mathrm{C}$ (Drummond et al., 1999). The presence of Chol has a relevant role in maintaining liposomal bilayer stability. The use of steric stabilization coatings makes the need for Chol less relevant for liposome assembly, although its use gains relevance for the assembly of drug-loaded liposomes, due to their capability to stabilize the drug inside the loaded liposomes.

In the Dex-loading liposome formulation optimization study, an inverse correlation of the system $P L$ and $E E$ with Chol concentration was observed (Table 2). The encapsulation of Dex was highest when no Chol was added to the liposome formulation. A possible explanation for this observation is the competitive encapsulation of Dex and Chol for the same sites within the liposome structure, with a clear advantage for Chol with respect to Dex. In this perspective, Dex is displaced from the positions in the membrane that are occupied by Chol. This may be related to the fact that the structures of the two compounds are very similar. This result is in accordance with other studies found in the literature (Tsotas et al., 2007; Bhardwaj and Burgess, 2010). The different stabilizing effects of Chol and Dex in the liposomes might be due to 
differences in their interaction with the phospholipid molecules. The highly lipophilic Chol is incorporated between acyl chains and reduces chain movement (increasing rigidity) above the phase transition temperature. Therefore, Chol shows membrane stabilization above the phase transition temperature. Dex, being more hydrophilic, may interact differently with the phospholipid acyl chains and head groups and may destabilize the membrane (Bhardwaj and Burgess, 2010).

The liposomes prepared in this study showed a monodisperse distribution. The $\zeta$ - potential increased for PEGylated liposomes (formulations E and F, Table 3). The $\zeta$-potential of liposomes is negative due to the presence of terminal carboxylic groups in the lipids. The value of the $\zeta$-potential for PEGylated liposomes was one order of magnitude lower, due to the negatively-charged phosphate group of DSPE-PEG, thus eliciting a reduced electrophoretic mobility and a high-energy barrier that avoids agglomeration and stabilizes the nanosuspension (Yousefi et al., 2008).

When liposomes hit a suitable and solid surface, they may adsorb, break and spread to form a bilayer on a hydrophilic surface or a monolayer on a hydrophobic one (Jass et al., 2000). An SEM image of non-coated liposomes prepared with formulation C (Figure1C) showed that the liposomes fused to each other, spread and flattened on the support surface. As the major structural components of cell membranes, phospholipids are critically important in membrane fusion processes. The bilayer structure and its physical properties (e.g. bilayer dehydration, lipid composition, lipid packing, bilayer curvature and non-bilayer phases) can contribute to membrane fusion. Hydration of the lipid head groups is also an important parameter in membrane fusion. These groups are hydrophilic and a shell of water is associated with the liposomal surface, representing a barrier to membrane fusion. Therefore, by removing the water from the head groups, the liposomes can lose their structures and fuse to each other. In conclusion, the incorporation of the DSPE-PEG in the liposomes helps to maintain the hydrated head groups, inducing the stabilization of the liposome structure.

An essential point in the evaluation of drug-delivery systems is the rate at which the drug is released from the carrier, predicting their in vivo behaviour. The release profile of Dex (Figure 2) showed an initial burst release, although Dex continued to be released at a slower but steady rate until day 21 . Similar results were obtained in other studies found in the literature (Tsotas et al., 2007; Bhardwaj and Burgess, 2010), in which Dex release kinetics from different types of liposomes were studied. It was found that Dex kinetic release is dependent on release, extrusion and lipid transition temperatures. Dex release from extruded liposomes was fast and most of the contents were released within $48 \mathrm{~h}$ at $37^{\circ} \mathrm{C}$. A slower release was found from DSPC which, according to the aforementioned studies, could be explained by the high transition temperature $\left(T_{c}=42^{\circ} \mathrm{C}\right)$. Indeed, in this study we also used HSPC in the liposome formulation, which has a $T_{c}=52^{\circ} \mathrm{C}$.

\subsection{Biological activity of Dex released from liposomes}

The osteogenic differentiation factor herein incorporated into the optimized liposomes formulation was previously studied by our group in different drug-release systems, including micro- and nanoparticles and nanofibrous and macroporous scaffolds (Balmayor et al., 2008, 2009b, Oliveira et al., 2008, 2009, 2010; Duarte et al., 2009). Herein, the effect of Dex-loaded liposomes on viability, proliferation, protein synthesis and osteogenic differentiation of hBMSCs was assessed by the use of standard research methods. Biological data show an earlier induction of hBMSCs differentiation into the osteogenic lineage, i.e. at day 7 of culture, as depicted by the results of ALP activity, osteoblastic protein expression and transcript expression patterns (i.e. immunocytochemistry and qPCR data). This observation is of significant relevance when compared with standard culture conditions for in vitro induction of hBMSCs osteogenic differentiation, demonstrating similar expression patterns to the TCPS_LipDex test condition for longer culture periods (i.e. for 14 and 21 days). This effect of time on the induction of osteogenic differentiation does not affect hBMSCs viability, demonstrating the absence of a toxicity effect by the Dex-loaded liposomes, but retards its proliferative capability, as depicted by the quantitative data from MTS and PicroGreen assays, respectively. Indeed, it is expected that an earlier differentiation may affect the proliferative potential of the cells.

Dexamethasone, besides being a differentiation factor, is also a well-known glucocorticoid, effectively used to attenuate inflammation in different clinical conditions, but with severe side-effects. Therefore, the local delivery of glucocorticoids by liposomes could be beneficial in various therapies (Asgeirdottir et al., 2007; Hegeman et al., 2011). Liposomes encapsulating this compound have shown to exert strong beneficial effects in inflammatory diseases (Hegeman et al., 2011), such as atherosclerosis (Chono and Morimoto, 2006) and osteoarthritis (Elron-Gross et al., 2009), and it has been evaluated in animal models of multiple sclerosis and Crohn's disease (Crielaard et al., 2011). The efficacy of Dex incorporated into liposomes in the treatment of atherosclerosis, the uptake of Dex-loaded liposomes by macrophages and foam cells and its inhibitory effect on cellular cholesterol ester accumulation in these cells have already been investigated in vitro (Chono and Morimoto, 2006). Besides the aforementioned studies, no studies have been published exploring these Dex-releasing biological nanoparticles or liposomes as a strategy to induce MSCs differentiation, in vitro or in vivo.

The approach described here constitutes an effective in vitro strategy to ameliorate the osteogenic differentiation of hBMSCs in time. Generally, under standard differentiation conditions, hBMSCs take 14-21 days to fully differentiate into the osteogenic lineage, which is time consuming (Jaiswal et al., 1997). With LipDex culture medium supplementation, the hBMSCs are differentiated 
into the osteogenic lineage at day 7 without compromising the viability and proliferation of the cells. Therefore, LipDex culture medium supplementation comprises a time-saving strategy when a stem cell-based therapy is envisioned for a predefined clinical situation. This timeeffective strategy of hBMSCs induction of differentiation could be transposed to other tissue-engineering and regenerative approaches, e.g. to the implantation of biofunctionalized scaffolds with surface-immobilized Dex-loaded liposomes, or by their direct injection at the injury site, to induce the differentiation of local or circulating MSCs in vivo. This is possible due to the capability of the liposomes to release this bioactive agent near to, or even internalize it into, the hBMSCs (Chono and Morimoto, 2006).

\section{Conclusion}

We propose liposomes as a differentiation factor release system to promote the osteogenesis of hBMSCs. The release profile of Dex showed an initial burst release, followed by a slower but steady release rate until day 21. We conclude that liposomes are non-cytotoxic. Our data shows that the release of Dex from liposomes promotes an earlier osteogenic differentiation of hBMSCs. The phenotypic and genotypic results are comparable. We believe that this result may have interesting implications for the development of cell-based therapies for bone regeneration in the context of autologous approaches.

\section{Acknowledgements}

The authors thank the Portuguese Foundation for Science and Technology for a PhD grant (No. SFRH/BD/62465/2009, to N. S. Monteiro). This work was partly supported by the FIND and BIND Project (No. NMP4-SL-2009-229292) and the OsteoGraphy Project (No. PTDC/EME-MFE/2008).

\section{Conflict of interest}

The authors have declared that there is no conflict of interest.

\section{References}

Allen TM, Hansen C, Martin F, et al. 1991; Liposomes containing synthetic lipid derivatives of poly(ethylene glycol) show prolonged circulation half-lives in vivo. Biochim Biophys Acta Biomemb 1066: 29-36.

Almeida AJ, Souto E. 2007; Solid lipid nanoparticles as a drug delivery system for peptides and proteins. Adv Drug Deliver Rev 59: 478-490.

Asgeirdottir SA, Kamps J, Bakker HI, et al. 2007; Site-specific inhibition of glomerulonephritis progression by targeted delivery of dexamethasone to glomerular endothelium. Mol Pharmacol 72: 121-131.

Balmayor ER, Feichtinger GA, Azevedo HS, et al. 2009a; Starch-poly( $\varepsilon$-caprolactone) microparticles reduce the needed amount of BMP-2. Clin Orthop Relat Res 467: 3138-3148.

Balmayor ER, Tuzlakoglu K, Azevedo HS, et al. 2009b; Preparation and characterization of starch-poly( $\varepsilon$-caprolactone) microparticles incorporating bioactive agents for drug delivery and tissue engineering applications. Acta Biomater 5: 1035-1045.

Balmayor ER, Tuzlakoglu K, Marques AP, et al. 2008; A novel enzymatically-mediated drug delivery carrier for bone tissue engineering applications: combining biodegradable starch-based microparticles and differentiation agents. J Mater Sci Mater Med 19: 1617-1623.

Bhardwaj U, Burgess DJ. 2010; Physicochemical properties of extruded and non-extruded liposomes containing the hydrophobic drug dexamethasone. Int J Pharm 388: 181-189.

Chen X, Huang W, Wong BCK, et al. 2012; Liposomes prolong the therapeutic effect of anti-asthmatic medication via pulmonary delivery. Int J Nanomed 7: 1139-1148.

Chono S, Morimoto K. 2006; Uptake of dexamethasone incorporated into liposomes by macrophages and foam cells and its inhibitory effect on cellular cholesterol ester accumulation. J Pharm Pharmacol 58: 1219-1225.

Chono S, Tauchi Y, Morimoto K. 2006; Influence of particle size on the distributions of liposomes to atherosclerotic lesions in mice. Drug Dev Ind Pharm 32: 125-135.

Crielaard BJ, Lammers T, Morgan ME, et al. 2011; Macrophages and liposomes in inflammatory disease: friends or foes? Int $J$ Pharm 416: 499-506.

Delorme B, Charbord P. 2007; Culture and characterization of human bone marrow mesenchymal stem cells. Methods Mol Med 140: 67-81.

Diebold Y, Jarrín M, Sáez V, et al. 2007; Ocular drug delivery by liposome-chitosan nanoparticle complexes (LCS-NP). Biomaterials 28: 1553-1564.

Drummond DC, Meyer O, Hong $\mathrm{K}$, et al. 1999; Optimizing liposomes for delivery of chemotherapeutic agents to solid tumors. Pharmacol Rev 51: 691-744.

Duarte ARC, Mano JF, Reis RL. 2009; Dexamethasone-loaded scaffolds prepared by supercritical-assisted phase inversion. Acta Biomater 5: 2054-2062.

Elron-Gross I, Glucksam Y, Margalit R. 2009; Liposomal dexamethasone-diclofenac combinations for local osteoarthritis treatment. Int J Pharm 376: 84-91.

Engler AJ, Sen S, Sweeney HL, et al. 2006; Matrix elasticity directs stem cell lineage specification. Cell 126: 677-689.

Gabizon A, Goren D, Cohen R, et al. 1998; Development of liposomal anthracyclines: from basics to clinical applications. J Control Release 53: 275-279.

Goldberg M, Langer R, Jia X. 2007; Nanostructured materials for applications in drug delivery and tissue engineering. J Biomater Sci Polym Ed 18: 241-268.
Gomes-da-Silva LC, Santos AO, Bimbo LM, et al. 2012; Toward a siRNA-containing nanoparticle targeted to breast cancer cells and the tumor microenvironment. Int $J$ Pharm 434: 9-19.

Hegeman MA, Cobelens PM, Kamps JAAM, et al. 2011; Liposome-encapsulated dexamethasone attenuates ventilator-induced lung inflammation. Br $J$ Pharmacol 163: 1048-1058.

Hristova K, Kenworthy A, McIntosh TJ. 1995; Effect of bilayer composition on the phase behavior of liposomal suspensions containing poly(ethylene glycol)-lipids. Macromolecules 28: 7693-7699.

Huang DM, Hung Y, Ko BS, et al. 2005; Highly efficient cellular labeling of mesoporous nanoparticles in human mesenchymal stem cells: implication for stem cell tracking. FASEB J 19: 2014-2016.

Jaiswal N, Haynesworth SE, Caplan AI, et al. 1997; Osteogenic differentiation of purified, culture-expanded human mesenchymal stem cells in vitro. $J$ Cell Biochem 64: 295-312.

Jass J, Tjarnhage T, Puu G. 2000; From liposomes to supported, planar bilayer structures on hydrophilic and hydrophobic surfaces: an atomic force microscopy study. Biophys $J$ 79: 3153-3163.

Kale AA, Torchilin VP. 2007; Enhanced transfection of tumor cells in vivo using 'Smart' pH-sensitive TAT-modified pegylated liposomes. J Drug Target 15: 538-545.

Katayama Y, Battista M, Kao WM, et al. 2006; Signals from the sympathetic nervous system regulate hematopoietic stem cell egress from bone marrow. Cell 124: 407-421.

Kim BYS, Rutka JT, Chan WCW. 2010; Nanomedicine. NEngl J Med 363: 2434-2443. Kulkarni M, Greiser U, O'Brien T, et al. 2010; Liposomal gene delivery mediated by 
tissue-engineered scaffolds. Trends Biotechnol 28: 28-36.

Lee SC, Lee KE, Kim JJ, et al. 2005; The effect of cholesterol in the liposome bilayer on the stabilization of incorporated retinol. $J$ Liposome Res 15: 157-166.

Leong-Poi H, Kuliszewski MA, Lekas M, et al. 2007; Therapeutic arteriogenesis by ultrasound-mediated VEGF165 plasmid gene delivery to chronically ischemic skeletal muscle. Circ Res 101: 295-303.

Li H, Song JH, Park JS, et al. 2003; Polyethylene glycol-coated liposomes for oral delivery of recombinant human epidermal growth factor. Int J Pharm 258: 11-19.

Liu N, Park HJ. 2010; Factors effect on the loading efficiency of vitamin C-loaded chitosancoated nanoliposomes. Colloids Surf $B$ Biointerfaces 76: 16-19.

Loughrey HC, Ferraretto A, Cannon AM, et al. 1993; Characterisation of biotinylated liposomes for in vivo targeting applications. FEBS Lett 332: 183-188.

Martins A, Duarte ARC, Faria S, et al. 2010; Osteogenic induction of hBMSCs by electrospun scaffolds with dexamethasone release functionality. Biomaterials 31: 5875-5885.

Mickova A, Buzgo M, Benada O, et al. 2012; Core/shell nanofibers with embedded liposomes as a drug delivery system. Biomacromolecules 13: 952-962.

Moreira JN, Ishida T, Gaspar R, et al. 2002; Use of the post-insertion technique to insert peptide ligands into pre-formed stealth liposomes with retention of binding activity and cytotoxicity. Pharm Res 19 265-269.
Moura V, Lacerda M, Figueiredo P, et al. 2012; Targeted and intracellular triggered delivery of therapeutics to cancer cells and the tumor microenvironment: impact on the treatment of breast cancer. Breast Cancer Res Treat 133: 61-73.

Oliveira JM, Kotobuki N, Marques AP, et al. 2008; Surface engineered carboxymethylchitosan/ poly(amidoamine) dendrimer nanoparticles for intracellular targeting. Adv Funct Mater 18: $1840-1853$.

Oliveira JM, Kotobuki N, Tadokoro M, et al. 2010; Ex vivo culturing of stromal cells with dexamethasone-loaded carboxymethylchitosan/ poly(amidoamine) dendrimer nanoparticles promotes ectopic bone formation. Bone 46: 1424-1435.

Oliveira JM, Sousa RA, Kotobuki N, et al. 2009; The osteogenic differentiation of rat bone marrow stromal cells cultured with dexamethasone-loaded carboxymethylchitosan/ poly(amidoamine) dendrimer nanoparticles. Biomaterials 30: 804-813.

Park JS, Yang HN, Woo DG, et al. 2011; The promotion of chondrogenesis, osteogenesis, and adipogenesis of human mesenchymal stem cells by multiple growth factors incorporated into nanosphere-coated microspheres. Biomaterials 32: 28-38.

Park TH, Lee HJ, Kim JA, et al. 2007; Application of magnetic bio-nanoparticles to the control of stem cell behavior. J Biotechnol 131: S65.

Pjanović R, Bošković-Vragolović N, VeljkovićGiga J, et al. 2010; Diffusion of drugs from hydrogels and liposomes as drug carriers. J Chem Technol Biotechnol 85: 693-698.
Santo VE, Duarte ARC, Gomes ME, et al. 2010; Hybrid 3D structure of poly(D,L-lactic acid) loaded with chitosan/chondroitin sulfate nanoparticles to be used as carriers for biomacromolecules in tissue engineering. J Supercrit Fluids 54: 320-327.

Shi J, Votruba AR, Farokhzad OC, et al. 2010; Nanotechnology in drug delivery and tissue engineering: from discovery to applications. Nano Lett 10: 3223-3230.

Song S, Liu D, Peng J, et al. 2009; Novel peptide ligand directs liposomes toward EGF-R high-expressing cancer cells in vitro and in vivo. FASEB J 23: 1396-1404.

Tan Q, Liu XJ, Fu XY, et al. 2012; Current development in nanoformulations of docetaxel. Expert Opin Drug Deliv 9: 975-990.

Torchilin VP, Weissig V. 2003; Liposomes: A Practical Approach. Oxford University Press: Oxford.

Tsotas VA, Mourtas S, Antimisiaris SG. 2007; Dexamethasone incorporating liposomes: effect of lipid composition on drug trapping efficiency and vesicle stability. Drug Deliv 14: 441-445.

Wu G, Mikhailovsky A, Khant HA, et al. 2008; Remotely triggered liposome release by nearinfrared light absorption via hollow gold nanoshells. J Am Chem Soc 130: 8175-8177.

Yousefi A, Esmaeili F, Rahimian S, et al. 2008; Preparation and in vitro evaluation of a pegylated nano-liposomal formulation containing docetaxel. Sci Pharm 77: 453-464.

Zheng X, Lu J, Deng L, et al. 2009; Preparation and characterization of magnetic cationic liposome in gene delivery. Int $J$ Pharm 366: 211-217. 